\title{
Antifungal activity of essential oils derived from some plants against phytopathogenic fungi \\ Habung Yami ${ }^{1}$ and A.K. Shukla ${ }^{2 *}$
}

${ }^{1}$ Department of Botany, Rajiv Gandhi University, Itanagar-791112, Arunachal Pradesh, India.

2Department of Botany, Indira Gandhi National Tribal University, Amarkantak-484887, Madhya Pradesh, India.

Received: June 27, 2016; Accepted: July 18, 2016.

\begin{abstract}
Essential oils were extracted from different plant species (Acorus calamus, Artemisia nilagerica, Erigeron Canadensis) to evaluate their effect on the growth of four phytopathogenic fungi viz. Alternaria alternata, Botrytis cinerea, Fusarium oxysporum and Penicillium expansum following poisoned food technique method. Different concentration of oil such as $125 \mathrm{ppm}, 250 \mathrm{ppm}, 500 \mathrm{ppm}, 1000 \mathrm{ppm}$ and $5000 \mathrm{ppm}$ were taken to evaluate the effect. There was $100 \%$ inhibition in the growth of phytopathogenic fungi at 5000 and $1000 \mathrm{ppm}$ concentration by essential oil of $A$. calamus. At $500 \mathrm{ppm}$ concentration also $100 \%$ inhibition was found up to $7^{\text {th }}$ day on $F$. oxysporum. Essential oil of $A$. nilagirica inhibits the growth of all phytopathogenic fungi at higher concentration. In case of P. expansum at $5000 \mathrm{ppm}$ concentration $100 \%$ inhibition was recorded even after 15 days of incubation. The decrease in colony diameter or growth of fungus was corresponding to the concentration of oil. In comparison to others two, essential oil of Erigeron Canadensis was less effective against phytopathogenic fungi. It can be stated on the basis of results that the use of Acorus calamus and Artemisia nilagirica essential oil could be an alternative to synthetic fungicides for management of post harvest phytopathogenic fungal diseases caused by $A$. alternata, $B$. cinerea, F. oxysporum and $P$. expansum.
\end{abstract}

Key words: Essential oil; fungitoxic; fungicidal; Phytopathogenic fungi; Acorus; Artemesia; Erigeron

\section{Introduction}

Essential oils or volatile oils are very complex mixture of compounds whose constituents of the oils are mainly monoterpenes and sesquiterpenes. Generally, the action of essential oils is the result of the combined effect of both their active and inactive compounds. These inactive compounds might influence resorption, rate of reactions and bioavailability of the active compounds. Until recently, essential oils have been studied most from the viewpoint of their flavor and fragrance only for flavoring foods, drinks and other goods. Actually, essential oils and their components are gaining increasing interest because of their relatively safe status, their wide acceptance by consumers, and their exploitation for potential multi-purpose functional use (Ormancey 2001).

Biologically active plant extracts, including essential oils, represent rich potential sources of alternative and perhaps environmentally more acceptable disease management compounds. Besides, higher plants also contain a wide spectrum of secondary substances viz. phenols, flavonoids, quinines, tannins, alkaloids, saponins and sterols. Plant diversity serves the humankind as renewable natural resources for a variety of biologically active chemicals. These chemicals bear a variety of properties viz. antibacterial, antifungal, antiviral, anthelmintic, anticancer, sedative, laxative, cardiotonic, diuretic and others (Parajuli et al., 1998). Naturally occurring biologically active compounds from plants are generally assumed to be more acceptable and less hazardous than synthetic compounds and represent a rich source of potential disease control agents. The secondary metabolites performs defensive role in plant from their invaders. The factors that affect biochemical profiles and secondary metabolite production in plant include physiological, genetics, and environmental variables. Active constituents of the medicinal and aromatic plants have been found to be less phytotoxic, more systemic and easily biodegradable (Fawcett and Spencer 1970).

The general antifungal activity of essential oils is well documented (Deans and Ritchie, 1987; Reuveni et.al., 1984; Tripathi and Shukla, 2007) and there have been some studies on the effects of essential oils on post harvest pathogens (Bishop and Thornton, 1997; Anthony et al, 2003; Tripathi et al., 2008). Biologically active essential oils represent a rich potential source of an alternative and perhaps environmentally more acceptable disease management compounds. There is need for an alternative approach to control phytopathonic fungi without toxicity problems that are ecofriendly and cost effective. Present study was aimed to evaluate the antifungal activity of some plant based essential oils against phytopathogenic fungi.

\footnotetext{
*Corresponding Author:

Prof. A. K. Shukla,

Department of Botany,

Indira Gandhi National Tribal University,

Amarkantak, Madhya Pradesh, India.

E-mail: ashukla21@,rediffmail.com
}

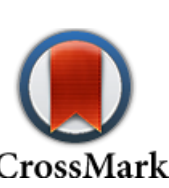


Materials and Methods

Isolation and identification
phytopathogenic fungi

of

Isolation of post harvest pathogens of kiwifruits were carried out from infected fruits on rose Bengal agar and potato dextrose agar medium (Johnson and Curl, 1972). Infected kiwifruits were randomly collected from market. Fruits were surface sterilized by $4 \%$ sodium hypochlorite and then by $75 \%$ alcohol and finally with sterilized distilled water. Small pieces of fruit were cut and placed in the petriplates containing sterilized medium and incubated at $27^{\circ} \mathrm{C}$ for $7-10$ days. Identification of fungal pathogens was done on the basis of morphological, cultural and microscopic characteristics as detailed in available literature (Barnett and Hunter 1972, Domsch et al., 1980). In process of culture the isolated fungal pathogens were cultivated on Potato Dextrose Agar (PDA) medium and Peptone Dextrose Rose Bengal Agar medium. Potato dextrose agar medium $(39 \mathrm{gm}$ of Hi-PDA medium dissolved in $1000 \mathrm{ml}$ of distilled water) medium was used throughout the investigation. The medium was autoclaved and cooled to $40^{\circ} \mathrm{C} \pm 2{ }^{\circ} \mathrm{C}$. Thirty milligram of streptomycin was added to it and mixed thoroughly so as to prevent bacterial contamination. Similarly, Peptone Dextrose Rose Bengal Agar (31.55 gm of Hi-RBA medium dissolved in $1000 \mathrm{ml}$ of distilled water) medium was prepared to maintain the fungal culture.

\section{Plant material collection and essential oils extraction}

Plants were collected from different parts of Arunachal Pradesh during the study period. Identification of plants was done by the plant taxonomist in the Department of Botany, Rajiv Gandhi University, Itanagar as well as by the Scientist from Regional Centre of Botanical Survey of India at Itanagar. Herbarium was preserved and voucher specimens were deposited in the department. Extraction of essential oils was carried out from some locally available larger number of angiospermic taxa namely Acorus calamus, Artimesia nilogerica, Erigeron canadensis, Eupatorium odoratum, Mesua ferrae, Mikania cordata, Piper mullesua and Pogostemon cablin etc. Subsequently on getting results potent 3 plants were taken for detailed study. An amount of $250 \mathrm{gm}$ of fresh leaves of each plant were cut separately into small pieces and were thoroughly washed with sterilized water. The volatile fractions were isolated by hydro distillation through Clevenger's apparatus. Leaves of the plants were used for extraction of essential oils except in case of Acorus calamus where fruits, rhizome and flowers were respectively used for the oil extraction. The isolated fractions of plant parts exhibited two distinct layers an upper oily layer and the lower aqueous layer. Both the layers were separated and the essential oils were stored in clean glass vials after removing water traces with the help of capillary tubes and anhydrous sodium sulphate (Guenther, 1972).

\section{Antifungal activity assay}

Fungitoxic activities of the essential oils were tested by the poisoned food technique of Grover and Moore (1962) and Perrucci et al., (1994). Potato dextrose agar medium (39 gm of Hi-PDA medium dissolved in $1000 \mathrm{ml}$ of distilled water) was used throughout the investigation. The medium was autoclaved and cooled to $40^{\circ} \mathrm{C} \pm 2^{\circ} \mathrm{C}$. Thirty milligram of streptomycin was added to it and mixed thoroughly so as to prevent bacterial contamination. A requisite amount of the oil was dissolved separately in $0.5 \mathrm{ml}$ of 0.01 percent of aqueous solution of Tween -80 in presterilized Petri plates $(7 \mathrm{~cm}$. diam.). While using Tween- 80 as solvent care was taken in designing the experiments to evaluate the true effect of essential oils on the pathogenic fungi. PDA medium (9.5 $\mathrm{ml}$ ) was pipetted to each Petri plate and was mixed so as to obtain the requisite concentrations viz. 5000ppm, 1000ppm, 500ppm, 250ppm and $125 \mathrm{ppm}$. For control sets, requisite amount of sterilized water in place of the oil was added to the medium.

Discs of test fungi (5 mm diam) were cut with the help of sterilized cork borer from the periphery of a seven-day old culture and were inoculated aseptically to the center of each petriplate of treatment and control sets. The petriplate were incubated at $27 \pm 1^{\circ} \mathrm{C}$ for six days in incubation chamber. Measurement of colony diameters of the test fungus in treatment and control sets were done in mutually perpendicular directions and were recorded in terms of percent mycelial inhibition using the following formula

Percentage of mycelial inhibition $=\frac{\mathrm{dc}-\mathrm{dt}}{\mathrm{dc}}$

Where $\mathrm{dc}=$ mean colony diameter of control sets

$\mathrm{dt}=$ mean colony diameter of treatment sets

Standardization of essential oils through fungitoxic properties

The standardization of essential oils was done through fungitoxic properties viz. minimum inhibitory concentration and nature of toxicity (Thompson, 1989).

\section{Minimum inhibitory concentration (MIC)}

To find out the minimum inhibitory concentration at which the oil showed absolute fungitoxicity (complete inhibition of growth of test fungi), experiments were carried out by the usual poisoned food technique. Different concentrations of the oils were prepared by dissolving separately their requisite amount in $0.5 \mathrm{ml}$ of 0.01 per cent of aqueous solution of Tween- 80 and then mixing with $9.5 \mathrm{ml}$ potato dextrose agar medium. The 
medium of control sets contained requisite amount of sterilized water dissolved in $0.5 \mathrm{ml}$ Tween- 80 in place of oils. As usual the prepared plates were inoculated upside down aseptically with the assay disc of the test fungi to the center of petriplate of treatment and control sets. The petriplates were incubated at $27 \pm 1^{\circ} \mathrm{C}$ for six days in BOD incubator. Diameters of fungal colony of treatment and control sets were measured in mutually perpendicular directions on the seventh day and percentage inhibition calculated.

\section{Nature of toxicity}

Nature of toxicity (funtgistatic / fungicidal) of essential oils against the fungi was determined as suggested by Thompson (1989). Requisite amount of the oil was dissolved separately in $0.5 \mathrm{ml}$ of 0.01 per cent of aqueous solution of Tween-80 and mixed with $9.5 \mathrm{ml}$ potato dextrose agar medium to get final concentrations. Sterilized water was used in control sets in place of the oils. The plates were inoculated upside down aseptically with fungal disc ( $5 \mathrm{~mm}$ diam.) taken from the periphery of a seven day old culture of the test fungi and were incubated for six days at $27 \pm 1^{\circ} \mathrm{C}$. On seventh day the inhibited discs were taken out from the plates, washed with sterilized water and reinoculated aseptically to plates containing fresh potato dextrose agar medium. The revival of the growth of the fungal discs was observed and the per cent inhibition of growth of the test fungi were calculated on the seventh day with respect to control sets.

\section{Results}

Evaluation of Essential Oils against Different Fungi

Essential oils extracted from different plant species were evaluated to visualize their effect on the growth of four phytopathogenic fungi viz. Penicillium expansum, Fusarium oxysporum, Botrytis cinerea and Alternaria alternata following Poisoned food technique method. Different concentration of oil such as $125 \mathrm{ppm}, 250 \mathrm{ppm}, 500 \mathrm{ppm}$, $1000 \mathrm{ppm}$ and $5000 \mathrm{ppm}$ were taken to evaluate the effect. Simultaneously, a control was also maintained by inoculating culture disc on the medium without adding any oil.

\section{Acorus calamus}

Essential oil of $A$. calamus was found effective against the growth of all tested fungi. In case of $P$. expansum and F. oxysporum 100\% inhibition of growth was recorded at 5000 and $1000 \mathrm{ppm}$ concentration by essential oil of $A$. calamus. At $500 \mathrm{ppm}$ concentration also 100\% inhibition was found up to $7^{\text {th }}$ day on F. oxysporum. But growth was observed during subsequent period of incubation. The decrease in colony diameter or growth of fungus was corresponding to the concentration of oil. On $A$. alternata and B. cinerea also the effect of oil was significant inhibitory. At 5000, 1000 and 500ppm concentration 100\% inhibition was recorded. At $250 \mathrm{ppm}$ also in case of A. alternata it restricts $100 \%$ up to $7^{\text {th }}$ day and after that slight growth was noticed during subsequent period of incubation.

Essential oil of $A$. calamus inhibited the growth of all four phytopathogenic fungi, at 5000, 1000 and $500 \mathrm{ppm}$ concentration and at lower level i.e 125 and 250ppm concentration of oil colony growth was recorded. But it always remains lesser than the control.

\section{Artemisia nilagirica}

Essential oil of $A$. nilagirica inhibits the growth of all phytopathogenic fungi at higher concentration. In case of $P$. expansum at $5000 \mathrm{ppm}$ concentration $100 \%$ inhibition was recorded even after 15 days of incubation. The decrease in colony diameter or growth of fungus was corresponding to the concentration of oil. On $A$. alternata and B. cinerea there was $100 \%$ inhibition up to $11^{\text {th }}$ day at $5000 \mathrm{ppm}$ concentration of oil. However, slight growth was seen on subsequent period of incubation. But in case of $F$. oxysporum even at $5000 \mathrm{ppm}$ concentration of oil slight growth was recorded. Increase in diameter of fungus colony was recorded at lower concentration of oil however it always remains lesser than control.

\section{Erigeron canadensis}

Essential oil of E. canadensis also inhibits the growth of phytopathogenic fungi. In case of $P$. expansum and F. oxysporum at higher concentration of oil inhibition was drastic. The decrease in colony diameter or growth of fungus was corresponding to the concentration of oil. While in case of $A$. alternata and $B$. cinerea at $5000 \mathrm{ppm}$ concentration of fungus colony was reduced initially but during subsequent period of incubation growth of colony increases but it always remain lesser than the control. At lower level of concentration of oil at $125 \mathrm{ppm}$ the growth of fungus colony was almost similar to the control. There was not much effect on growth of fungus colony by E. canadensis oil.

\section{Minimum Inhibitory concentration (MIC)}

Inhibitory evaluation of essential oils against phytopathogenic fungi showed the effective results. Essential oil of Acorus calamus was found fungi toxic at $250 \mathrm{ppm}$ for $A$. alternata and $500 \mathrm{ppm}$ for rest of the three fungi. EO of Erigeron canadensis was inhibitory at higher concentration for all the phytopathogenic fungi. Artemisia nilagirica $\mathrm{EO}$ was found fungitoxic at $5000 \mathrm{ppm}$ concentration against $A$. alternata $B$. cinerea, and $P$. expansum. 


\section{Nature of toxicity}

Acorus calamus oil was found fungicidal for all the phytopathogenic fungi. Oil of E. Canadensis was fungistatic for all the pathogens. Artemisia nilogirica oil was fungicidal at $500 \mathrm{ppm}$ for all the tested fungi except $F$. oxysporum for which it was fungistatic.

Table 1: Effect of Acorus calamus essential oil on the phytopathogenic fungi

\begin{tabular}{|c|c|c|c|c|c|c|}
\hline Period & \multicolumn{6}{|c|}{ Alternaria alternata } \\
\hline Days & $5000 \mathrm{ppm}$ & 1000ppm & 500ppm & 250ppm & 125ppm & Control \\
\hline $5^{\text {th }}$ & $0.00 \pm 0.00$ & $0.00 \pm 0.00$ & $0.00 \pm 0.00$ & $0.00 \pm 0.00$ & $1.10 \pm 0.00$ & $2.60 \pm 0.00$ \\
\hline $7^{\text {th }}$ & $0.00 \pm 0.00$ & $0.00 \pm 0.00$ & $0.00 \pm 0.00$ & $0.00 \pm 0.00$ & $1.65 \pm 0.05$ & $3.20 \pm 0.10$ \\
\hline $9^{\text {th }}$ & $0.00 \pm 0.00$ & $0.00 \pm 0.00$ & $0.00 \pm 0.00$ & $0.50 \pm 0.00$ & $2.05 \pm 0.05$ & $4.90 \pm 0.10$ \\
\hline $11^{\text {th }}$ & $0.00 \pm 0.00$ & $0.00 \pm 0.00$ & $0.00 \pm 0.00$ & $0.90 \pm 0.40$ & $2.60 \pm 0.00$ & $5.60 \pm 0.10$ \\
\hline $13^{\text {th }}$ & $0.00 \pm 0.00$ & $0.00 \pm 0.00$ & $0.00 \pm 0.00$ & $2.05 \pm 0.25$ & $3.15 \pm 0.05$ & $6.00 \pm 0.10$ \\
\hline \multirow[t]{2}{*}{$15^{\text {th }}$} & $0.00 \pm 0.00$ & $0.00 \pm 0.00$ & $0.00 \pm 0.00$ & $2.45 \pm 0.25$ & $3.80 \pm 0.00$ & $6.20 \pm 0.10$ \\
\hline & \multicolumn{6}{|c|}{ Botrytis cinerea } \\
\hline Days & $5000 \mathrm{ppm}$ & $1000 \mathrm{ppm}$ & $500 \mathrm{ppm}$ & $250 \mathrm{ppm}$ & $125 \mathrm{ppm}$ & Control \\
\hline $5^{\text {th }}$ & $0.00 \pm 0.00$ & $0.00 \pm 0.00$ & $0.00 \pm 0.00$ & $1.65 \pm 1.50$ & $3.05 \pm 2.80$ & $4.50 \pm 0.10$ \\
\hline $7^{\text {th }}$ & $0.00 \pm 0.00$ & $0.00 \pm 0.00$ & $0.00 \pm 0.00$ & $2.50 \pm 0.30$ & $4.25 \pm 0.65$ & $5.90 \pm 0.00$ \\
\hline $9^{\text {th }}$ & $0.00 \pm 0.00$ & $0.00 \pm 0.00$ & $0.00 \pm 0.00$ & $2.85 \pm 0.35$ & $4.70 \pm 0.80$ & $6.50 \pm 0.10$ \\
\hline $11^{\text {th }}$ & $0.00 \pm 0.00$ & $0.00 \pm 0.00$ & $0.00 \pm 0.00$ & $4.25 \pm 0.45$ & $4.85 \pm 0.85$ & $6.80 \pm 0.10$ \\
\hline $13^{\text {th }}$ & $0.00 \pm 0.00$ & $0.00 \pm 0.00$ & $0.00 \pm 0.00$ & $4.60 \pm 0.30$ & $5.10 \pm 0.60$ & $6.80 \pm 0.10$ \\
\hline \multirow[t]{2}{*}{$15^{\text {th }}$} & $0.00 \pm 0.00$ & $0.00 \pm 0.00$ & $0.00 \pm 0.00$ & $4.90 \pm 0.00$ & $5.10 \pm 0.60$ & $7.00 \pm 0.10$ \\
\hline & \multicolumn{6}{|c|}{ Fusarium oxysporum } \\
\hline Days & $5000 \mathrm{ppm}$ & 1000ppm & 500ppm & $250 \mathrm{ppm}$ & 125ppm & Control \\
\hline $5^{\text {th }}$ & $0.00 \pm 0.00$ & $0.00 \pm 0.00$ & $0.00 \pm 0.00$ & $0.90 \pm 0.40$ & $1.65 \pm 0.15$ & $4.00 \pm 0.00$ \\
\hline $7^{\text {th }}$ & $0.00 \pm 0.00$ & $0.00 \pm 0.00$ & $0.00 \pm 0.00$ & $1.75 \pm 0.25$ & $2.50 \pm 0.00$ & $4.50 \pm 0.10$ \\
\hline $9^{\text {th }}$ & $0.00 \pm 0.00$ & $0.00 \pm 0.00$ & $0.75 \pm 0.25$ & $2.35 \pm 0.25$ & $3.30 \pm 0.20$ & $4.70 \pm 0.10$ \\
\hline $11^{\text {th }}$ & $0.00 \pm 0.00$ & $0.00 \pm 0.00$ & $1.45 \pm 0.05$ & $3.2 \pm 0.30$ & $4.15 \pm 0.35$ & $5.40 \pm 0.10$ \\
\hline $13^{\text {th }}$ & $0.00 \pm 0.00$ & $0.00 \pm 0.00$ & $2.05 \pm 0.05$ & $4.15 \pm 0.15$ & $5.20 \pm 0.40$ & $6.30 \pm 0.10$ \\
\hline \multirow[t]{2}{*}{$15^{\text {th }}$} & $0.00 \pm 0.00$ & $0.00 \pm 0.00$ & $2.65 \pm 0.05$ & $5.05 \pm 0.25$ & $5.95 \pm 0.25$ & $7.00 \pm 0.10$ \\
\hline & \multicolumn{6}{|c|}{ Penicillium expensum } \\
\hline Days & $5000 \mathrm{ppm}$ & $1000 \mathrm{ppm}$ & $500 \mathrm{ppm}$ & $250 \mathrm{ppm}$ & $125 \mathrm{ppm}$ & Control \\
\hline $5^{\text {th }}$ & $0.00 \pm 0.00$ & $0.00 \pm 0.00$ & $0.00 \pm 0.00$ & $0.50 \pm 0.00$ & $1.20 \pm 0.10$ & $3.60 \pm 0.10$ \\
\hline $7^{\text {th }}$ & $0.00 \pm 0.00$ & $0.00 \pm 0.00$ & $0.00 \pm 0.00$ & $1.00 \pm 0.00$ & $1.95 \pm 0.05$ & $4.50 \pm 0.10$ \\
\hline $9^{\text {th }}$ & $0.00 \pm 0.00$ & $0.00 \pm 0.00$ & $0.00 \pm 0.00$ & $1.45 \pm 0.05$ & $2.65 \pm 0.05$ & $5.00 \pm 0.10$ \\
\hline $11^{\text {th }}$ & $0.00 \pm 0.00$ & $0.00 \pm 0.00$ & $0.50 \pm 0.00$ & $1.95 \pm 0.05$ & $3.15 \pm 0.15$ & $5.50 \pm 0.10$ \\
\hline $13^{\text {th }}$ & $0.00 \pm 0.00$ & $0.00 \pm 0.00$ & $0.75 \pm 0.05$ & $1.95 \pm 0.05$ & $3.85 \pm 0.05$ & $5.80 \pm 0.10$ \\
\hline $15^{\text {th }}$ & $0.00 \pm 0.00$ & $0.00 \pm 0.00$ & $0.75 \pm 0.05$ & $2.15 \pm 0.15$ & $4.15 \pm 0.15$ & $6.00 \pm 0.10$ \\
\hline
\end{tabular}

Table 2: Effect of Artemisia nilagerica essential oil on the phytopathogenic fungi

\begin{tabular}{|c|c|c|c|c|c|c|}
\hline Period & \multicolumn{6}{|c|}{ Alternaria alternata } \\
\hline Days & 5000ppm & 1000ppm & 500ppm & $250 \mathrm{ppm}$ & 125ppm & Control \\
\hline $5^{\text {th }}$ & $0.00 \pm 0.00$ & $0.00 \pm 0.00$ & $2.60 \pm 0.20$ & $3.35 \pm 0.05$ & $3.90 \pm 0.10$ & $2.60 \pm 0.00$ \\
\hline $7^{\text {th }}$ & $0.00 \pm 0.00$ & $0.00 \pm 0.00$ & $3.25 \pm 0.55$ & $3.05 \pm 0.05$ & $4.50 \pm 0.10$ & $3.20 \pm 0.10$ \\
\hline $9^{\text {th }}$ & $0.00 \pm 0.00$ & $0.00 \pm 0.00$ & $3.25 \pm 0.55$ & $4.15 \pm 0.05$ & $5.15 \pm 0.15$ & $4.90 \pm 0.10$ \\
\hline $11^{\text {th }}$ & $0.00 \pm 0.00$ & $0.00 \pm 0.00$ & $3.25 \pm 0.55$ & $5.00 \pm 0.10$ & $5.25 \pm 0.25$ & $5.60 \pm 0.10$ \\
\hline $13^{\text {th }}$ & $0.00 \pm 0.00$ & $0.00 \pm 0.00$ & $3.30 \pm 0.50$ & $5.00 \pm 0.10$ & $5.60 \pm 0.20$ & $6.00 \pm 0.10$ \\
\hline \multirow[t]{2}{*}{$15^{\text {th }}$} & $0.00 \pm 0.00$ & $0.00 \pm 0.00$ & $3.30 \pm 0.50$ & $5.00 \pm 0.10$ & $5.60 \pm 0.20$ & $6.20 \pm 0.10$ \\
\hline & \multicolumn{6}{|c|}{ Botrytis cinerea } \\
\hline Days & $5000 \mathrm{ppm}$ & 1000ppm & $500 \mathrm{ppm}$ & $250 \mathrm{ppm}$ & $125 \mathrm{ppm}$ & Control \\
\hline 5 th & $0.00 \pm 0.00$ & $0.00 \pm 0.00$ & $2.6 \pm 0.25$ & $3.35 \pm 0.05$ & $3.90 \pm 0.00$ & $4.50 \pm 0.10$ \\
\hline 7 th & $0.00 \pm 0.00$ & $0.00 \pm 0.00$ & $3.25 \pm 0.6$ & $4.05 \pm 0.20$ & $4.50 \pm 0.15$ & $5.90 \pm 0.00$ \\
\hline 9th & $0.00 \pm 0.00$ & $0.00 \pm 0.00$ & $3.25 \pm 0.6$ & $4.15 \pm 0.10$ & $5.15 \pm 0.25$ & $6.50 \pm 0.10$ \\
\hline 11th & $0.00 \pm 0.00$ & $0.00 \pm 0.00$ & $3.25 \pm 0.6$ & $5.00 \pm 0.30$ & $5.25 \pm 0.20$ & $6.80 \pm 0.10$ \\
\hline 13th & $0.00 \pm 0.00$ & $0.00 \pm 0.00$ & $3.3 \pm 0.55$ & $5.00 \pm 0.30$ & $5.60 \pm 0.20$ & $6.80 \pm 0.10$ \\
\hline \multirow[t]{2}{*}{15 th } & $0.00 \pm 0.00$ & $0.00 \pm 0.00$ & $3.3 \pm 0.65$ & $5.00 \pm 0.30$ & $5.60 \pm 0.20$ & $7.00 \pm 0.10$ \\
\hline & \multicolumn{6}{|c|}{ Fusarium oxysporum } \\
\hline Days & 5000ppm & 1000ppm & $500 \mathrm{ppm}$ & $250 \mathrm{ppm}$ & $125 \mathrm{ppm}$ & Control \\
\hline $5^{\text {th }}$ & $0.00 \pm 0.00$ & $0.00 \pm 0.00$ & $1.75 \pm 0.05$ & $2.00 \pm 0.00$ & $2.10 \pm 0.10$ & $4.00 \pm 0.00$ \\
\hline $7^{\text {th }}$ & $0.00 \pm 0.00$ & $0.00 \pm 0.00$ & $2.15 \pm 0.05$ & $2.35 \pm 0.05$ & $2.50 \pm 0.10$ & $4.50 \pm 0.10$ \\
\hline $9^{\text {th }}$ & $0.00 \pm 0.00$ & $0.00 \pm 0.00$ & $2.45 \pm 0.05$ & $2.75 \pm 0.05$ & $2.90 \pm 0.10$ & $4.70 \pm 0.10$ \\
\hline 11th & $0.00 \pm 0.00$ & $0.00 \pm 0.00$ & $2.90 \pm 0.00$ & $2.75 \pm 0.05$ & $2.90 \pm 0.10$ & $5.40 \pm 0.10$ \\
\hline $13^{\text {th }}$ & $0.00 \pm 0.00$ & $0.00 \pm 0.00$ & $3.35 \pm 0.05$ & $3.50 \pm 0.00$ & $3.60 \pm 0.10$ & $6.30 \pm 0.10$ \\
\hline \multirow[t]{2}{*}{$15^{\text {th }}$} & $0.00 \pm 0.00$ & $0.00 \pm 0.00$ & $3.35 \pm 0.05$ & $4.00 \pm 0.00$ & $4.25 \pm 0.25$ & $7.00 \pm 0.10$ \\
\hline & \multicolumn{6}{|c|}{ Penicillium expensum } \\
\hline Days & $5000 \mathrm{ppm}$ & 1000ppm & 500ppm & $250 \mathrm{ppm}$ & $125 \mathrm{ppm}$ & Control \\
\hline 5 th & $0.00 \pm 0.00$ & $0.00 \pm 0.00$ & $1.20 \pm 0.10$ & $3.40 \pm 0.20$ & $3.75 \pm 0.05$ & $3.60 \pm 0.10$ \\
\hline 7 th & $0.00 \pm 0.00$ & $0.00 \pm 0.00$ & $2.05 \pm 0.05$ & $3.80 \pm 0.20$ & $4.00 \pm 0.00$ & $4.50 \pm 0.10$ \\
\hline 9th & $0.00 \pm 0.00$ & $0.00 \pm 0.00$ & $2.55 \pm 0.25$ & $4.10 \pm 0.10$ & $4.35 \pm 0.05$ & $5.00 \pm 0.10$ \\
\hline 11th & $0.00 \pm 0.00$ & $0.00 \pm 0.00$ & $2.55 \pm 0.25$ & $4.10 \pm 0.10$ & $5.05 \pm 0.05$ & $5.50 \pm 0.10$ \\
\hline 13th & $0.00 \pm 0.00$ & $0.00 \pm 0.00$ & $2.75 \pm 0.25$ & $4.35 \pm 0.15$ & $5.05 \pm 0.05$ & $5.80 \pm 0.10$ \\
\hline 15 th & $0.00 \pm 0.00$ & $2.05 \pm 0.05$ & $2.75 \pm 0.25$ & $4.35 \pm 0.15$ & $5.15 \pm 0.05$ & $6.00 \pm 0.10$ \\
\hline
\end{tabular}


Table 3: Effect of Erigeron Canadensis essential oil on the phytopathogenic fungi.

\begin{tabular}{|c|c|c|c|c|c|c|}
\hline Period & \multicolumn{6}{|c|}{ Alternaria alternata } \\
\hline Days & $5000 \mathrm{ppm}$ & $1000 \mathrm{ppm}$ & 500ppm & 250ppm & 125ppm & Control \\
\hline $5^{\text {th }}$ & $0.00 \pm 0.00$ & $4.05 \pm 0.25$ & $4.15 \pm 0.05$ & $4.20 \pm 0.10$ & $4.25 \pm 0.05$ & $2.60 \pm 0.00$ \\
\hline $7^{\text {th }}$ & $0.00 \pm 0.00$ & $4.45 \pm 0.55$ & $4.70 \pm 0.10$ & $4.75 \pm 0.15$ & $5.20 \pm 0.40$ & $3.20 \pm 0.10$ \\
\hline $9^{\text {th }}$ & $0.00 \pm 0.00$ & $4.70 \pm 0.80$ & $4.95 \pm 0.25$ & $4.95 \pm 0.05$ & $6.10 \pm 0.40$ & $4.90 \pm 0.10$ \\
\hline $11^{\text {th }}$ & $4.10 \pm 0.90$ & $4.75 \pm 0.75$ & $5.00 \pm 0.20$ & $4.95 \pm 0.05$ & $6.10 \pm 0.40$ & $5.60 \pm 0.10$ \\
\hline $13^{\text {th }}$ & $4.10 \pm 0.90$ & $4.75 \pm 0.75$ & $5.00 \pm 0.20$ & $4.95 \pm 0.05$ & $6.10 \pm 0.40$ & $6.00 \pm 0.10$ \\
\hline \multirow[t]{2}{*}{$15^{\text {th }}$} & $4.10 \pm 0.90$ & $4.75 \pm 0.75$ & $5.00 \pm 0.20$ & $4.95 \pm 0.05$ & $6.10 \pm 0.40$ & $6.20 \pm 0.10$ \\
\hline & \multicolumn{6}{|c|}{ Botrytis cinerea } \\
\hline Days & $5000 \mathrm{ppm}$ & $1000 \mathrm{ppm}$ & $500 \mathrm{ppm}$ & $250 \mathrm{ppm}$ & $125 \mathrm{ppm}$ & Control \\
\hline $5^{\text {th }}$ & $0.00 \pm 0.00$ & $4.05 \pm 0.25$ & $4.30 \pm 0.20$ & $3.95 \pm 0.15$ & $3.95 \pm 0.65$ & $4.50 \pm 0.10$ \\
\hline $7^{\text {th }}$ & $0.00 \pm 0.00$ & $4.70 \pm 0.80$ & $4.70 \pm 0.10$ & $5.25 \pm 0.45$ & $4.10 \pm 0.80$ & $5.90 \pm 0.00$ \\
\hline $9^{\text {th }}$ & $0.00 \pm 0.00$ & $4.95 \pm 1.05$ & $4.95 \pm 0.25$ & $6.10 \pm 0.40$ & $4.20 \pm 0.70$ & $6.50 \pm 0.10$ \\
\hline $11^{\text {th }}$ & $0.00 \pm 0.00$ & $4.95 \pm 1.05$ & $4.95 \pm 0.25$ & $6.10 \pm 0.40$ & $4.20 \pm 0.70$ & $6.80 \pm 0.10$ \\
\hline $13^{\text {th }}$ & $4.10 \pm 0.90$ & $4.95 \pm 1.05$ & $4.95 \pm 0.25$ & $6.10 \pm 0.40$ & $4.20 \pm 0.70$ & $6.80 \pm 0.10$ \\
\hline \multirow[t]{2}{*}{$15^{\text {th }}$} & $4.10 \pm 0.90$ & $4.95 \pm 1.05$ & $4.95 \pm 0.25$ & $6.10 \pm 0.40$ & $4.20 \pm 0.70$ & $7.00 \pm 0.10$ \\
\hline & \multicolumn{6}{|c|}{ Fusarium oxysporum } \\
\hline Days & $5000 \mathrm{ppm}$ & $1000 \mathrm{ppm}$ & $500 \mathrm{ppm}$ & $250 \mathrm{ppm}$ & $125 \mathrm{ppm}$ & Control \\
\hline $5^{\text {th }}$ & $0.00 \pm 0.00$ & $1.10 \pm 0.10$ & $1.45 \pm 0.05$ & $2.10 \pm 0.10$ & $2.25 \pm 0.25$ & $4.00 \pm 0.00$ \\
\hline $7^{\text {th }}$ & $0.00 \pm 0.00$ & $1.10 \pm 0.10$ & $1.90 \pm 0.10$ & $2.50 \pm 0.10$ & $2.80 \pm 0.20$ & $4.50 \pm 0.10$ \\
\hline $9^{\text {th }}$ & $1.15 \pm 0.05$ & $1.60 \pm 0.10$ & $2.20 \pm 0.10$ & $2.95 \pm 0.05$ & $3.30 \pm 0.30$ & $4.70 \pm 0.10$ \\
\hline $11^{\text {th }}$ & $1.50 \pm 0.10$ & $1.90 \pm 0.10$ & $2.55 \pm 0.05$ & $3.50 \pm 0.10$ & $3.65 \pm 0.15$ & $5.40 \pm 0.10$ \\
\hline $13^{\text {th }}$ & $1.75 \pm 0.05$ & $2.20 \pm 0.10$ & $2.95 \pm 0.05$ & $3.90 \pm 0.10$ & $4.05 \pm 0.15$ & $6.30 \pm 0.10$ \\
\hline \multirow[t]{2}{*}{$15^{\text {th }}$} & $2.05 \pm 0.05$ & $2.45 \pm 0.05$ & $3.25 \pm 0.25$ & $4.25 \pm 0.05$ & $4.50 \pm 0.10$ & $7.00 \pm 0.10$ \\
\hline & \multicolumn{6}{|c|}{ Penicillium expensum } \\
\hline Days & $5000 \mathrm{ppm}$ & 1000ppm & $500 \mathrm{ppm}$ & $250 \mathrm{ppm}$ & $125 \mathrm{ppm}$ & Control \\
\hline $5^{\text {th }}$ & $0.00 \pm 0.00$ & $1.65 \pm 0.05$ & $2.00 \pm 0.10$ & $2.30 \pm 0.10$ & $3.05 \pm 0.05$ & $3.60 \pm 0.10$ \\
\hline $7^{\text {th }}$ & $0.00 \pm 0.00$ & $1.95 \pm 0.05$ & $2.35 \pm 0.05$ & $2.55 \pm 0.05$ & $3.65 \pm 0.65$ & $4.50 \pm 0.10$ \\
\hline $9^{\text {th }}$ & $2.20 \pm 0.10$ & $2.25 \pm 0.05$ & $2.75 \pm 0.05$ & $2.90 \pm 0.10$ & $3.95 \pm 0.75$ & $5.00 \pm 0.10$ \\
\hline $11^{\text {th }}$ & $2.60 \pm 0.10$ & $2.65 \pm 0.05$ & $3.05 \pm 0.05$ & $2.90 \pm 0.10$ & $4.25 \pm 0.75$ & $5.50 \pm 0.10$ \\
\hline $13^{\text {th }}$ & $3.05 \pm 0.05$ & $3.00 \pm 0.10$ & $3.05 \pm 0.05$ & $3.15 \pm 0.15$ & $4.50 \pm 0.50$ & $5.80 \pm 0.10$ \\
\hline $15^{\text {th }}$ & $3.05 \pm 0.05$ & $3.05 \pm 0.05$ & $3.25 \pm 0.05$ & $3.15 \pm 0.15$ & $4.50 \pm 0.50$ & $6.00 \pm 0.10$ \\
\hline
\end{tabular}

Table 4. Minimum inhibitory concentration of essential oils against pathogenic fungi

\begin{tabular}{lllll}
\hline \multirow{2}{*}{ Essential oils of plants } & \multicolumn{4}{c}{ MIC of oils against fungi } \\
\cline { 2 - 5 } & A. alternata & B. cinerea & $\boldsymbol{F}$. oxysporum & $\boldsymbol{P}$. expansum \\
\hline Acorus calamus & $250 \mathrm{ppm}$ & $500 \mathrm{ppm}$ & $500 \mathrm{ppm}$ & $500 \mathrm{ppm}$ \\
Artemisia nilagerica & $5000 \mathrm{ppm}$ & $5000 \mathrm{ppm}$ & Higher Conc. & $5000 \mathrm{ppm}$ \\
Erigeron canadensis & Higher Conc. & Higher Conc. & Higher Conc. & Higher Conc. \\
\hline
\end{tabular}

Table 5. Toxicity nature of Essential oils on phytopathogenic fungi

\begin{tabular}{lllll}
\hline Essential oils & \multicolumn{1}{c}{$\boldsymbol{A}$. alternata } & \multicolumn{1}{c}{ B. cinerea } & \multicolumn{1}{c}{ F. oxysporum } & \multicolumn{1}{c}{. expansum } \\
\hline Acorus calamus & Fungicidal at $500 \mathrm{ppm}$ & Fungicidal at $500 \mathrm{ppm}$ & Fungicidal at $1000 \mathrm{ppm}$ & Fungicidal at $500 \mathrm{ppm}$ \\
Artemisia nilogirica & Fungicidal at $500 \mathrm{ppm}$ & Fungicidal at $500 \mathrm{ppm}$ & Fungistatic & Fungicidal at $5000 \mathrm{ppm}$ \\
Erigeron canadensis & Fungistatic & Fungistatic & Fungistatic & Fungistatic \\
\hline
\end{tabular}

\section{Discussion}

Essential oil of $A$. calamus was recorded inhibitory for the growth of all tested fungi. Colony growth of P. expansum and F. oxysporum restricted $100 \%$ at 5000 and $1000 \mathrm{ppm}$ concentration of $A$. calamus essential oil. Colony growth of A. alternata and $B$. cinerea was also inhibited by oil and at 5000, 1000 and $500 \mathrm{ppm}$ concentration $100 \%$ inhibition was recorded. Results indicate that $A$. calamus essential oil have fungitoxic and fungistatic property against the phytopathogenic fungi. Mazza (1985) found, that Indian calamus oil contained high amount of $\beta$-asarone $(77.7 \%)$ and $6.8 \% \quad \alpha$-asarone, but in European calamus oil acorenone (8.1\%), isoshyobunone $(6.3 \%), \quad \beta$-gurjunene $(6.7 \%)$, calamendiol $(5.2 \%)$ and $\beta$-asarone $(5.2 \%)$ were found to be major components. The complexity in essential oils is due to terpene hydrocarbons as well as their oxygenated derivatives, such as alcohols, aldehydes, ketones, acids and esters (Wijesekara et al., 1997). Radusiene (2007) reported that essential oils of $A$. calamus were dominated by the presence of phenolic compounds: $(Z)$-asarone $(15.7-25.5 \%)$ and (Z)-methyl isoeugenol (2.0 $4.9 \%$ ). Other identified major components were (E)-caryophyllene, $\alpha$-humulene, germacrene, linalool, camphor and isoborneol. Satyal (2013) isolated a number of compounds from the essential oil of $A$. calamus and noted cytotoxicity and antifungal activity against Aspergillus niger. Sharma et al., (2007) reported antifungal activity of Acorus calamus oil against Sclerotium rolfsii and Rbizoctonia bataticola. Devi and Ganjewala (2009) found remarkable antifungal activity of $A$. calamus oil against Aspergillus niger, A. flavus, Microsporum canis and Penicillium chrysogenum. Lee et al., (2007) attributed antifungal activity of $\alpha$-asarone and aldehydes present in the $A$. calamus oil. Due to presence of a number of compounds and high quantity of phenolics in essential oil of $A$. calamus, perhaps any one of that or in combination would 
have inhibited the colony growth of the tested phytopathogenic fungi.

Essential oil of $A$. nilagerica inhibits the growth of all phytopathogenic fungi at higher concentration. In case of $P$. expansum at $5000 \mathrm{ppm}$ concentration $100 \%$ inhibition was recorded even after 15 days of incubation. The decrease in colony diameter or growth of fungus was corresponding to the concentration of oil. On $A$. alternata and B. cinerea there was $100 \%$ inhibition up to 11 th day at $5000 \mathrm{ppm}$ concentration of oil. However, slight growth was seen on subsequent period of incubation. At low concentration nature of oil was fungistatic. Sati et al., (2013) reported that essential oil contained approximately $79.91 \%$ monoterpenoids and $18.25 \%$ sesquiterpenoids. Thujone $(36.35 \%)$, -thujone $(9.37 \%)$, germacrene D $(6.32 \%)$, 4-terpineol (6.31\%), -caryophyllene $(5.43 \%)$, camphene $(5.47 \%)$ and borneol $(4.12 \%)$ as the major constituents. The essential oil exhibited significant antifungal activity against Rhizoctonia solani (ED50, $85.75 \mathrm{mg}$ L1), Sclerotium rolfsii (ED50, $87.63 \mathrm{mg} \mathrm{L1}$ ) and Macrophomina phaseolina (ED50, $93.23 \mathrm{mg}$ L1). Padalia et al., (2014) found that essential oils were mainly composed of monoterpenoids (59.0\%-77.3\%) and sesquiterpenoids (15.7\%-31.6\%). The major constituents identified were artemisia ketone (38.3\%-61.2\%), chrysanthenone (1.5\%-7.7\%), germacrene D $(3.1 \%-6.8 \%), \quad \beta$-caryophyllene (1.9\%-6.8\%), germacra-4,5, 10-trien-1- $\alpha$-ol $(1.9 \%$ $4.9 \%)$ and artemisia alcohol (1.4\%-3.6\%). Stappen et al., (2014) reported that $A$. nilagirica essential oil have nonselective antifungal activity against plant pathogens Colletotrichum acutatum, Colletotrichum fragariae and Colletotrichum gloeosporioides. Presence of terpenoides in large quantity and other compounds in small quantity would have perhaps played antifungal property against the phytopathogenic fungi.

Essential oil of Erigeron canadensis also inhibits the growth of phytopathogenic fungi $P$. expansum and F. oxysporum at higher concentration. The decrease in colony diameter or growth of fungus was corresponding to the concentration of oil. While in case of $A$. alternata and $B$. cinerea at $5000 \mathrm{ppm}$ concentration of fungus colony was reduced initially but during subsequent period of incubation growth of colony increases but it always remain lesser than the control. Unnithan (2014) reported in the essential oil of Erigeron canadensis a total of 23 components and main constituents were monoterpenoids (limomene 57.2\%), camphene $(2.5 \%) \alpha$ and $\beta$-pinenes $(1.9 \% \& 2.1 \%)$ and sesquiterpenoids (caryophyllene (6.7\%), germacrene D (4.9\%) and $\alpha$-curcumene $(3.0 \%)$. A few non-terpenoid acetylenic compounds (4.8\%) were also detected. Curini et al., (2003) found essential oils of $E$. canadensis under in vitro condition as growth inhibitors against phytopathogenic fungi Rhizoctonia solani Kuhn, Fusarium solani and Colletotrichum lindemutbianum but with weak fungicidal acitivity.

It can be concluded on the basis of present findings that the use of Acorus calamus and Artemisia nilagirica essential oil could be an alternative to synthetic fungicides for management of postharvest phytopathogenic fungal diseases caused by A. alternata, B. cinerea, F. oxysporum and P. expansum.

\section{References}

1. Anthony S, Abeywickrama K and Wilson Wijeratnam S. The effect of spraying essential oils of Cymbopogon nardus, Cymbopogon flexuosus and Ocimum basilicum on postharvest diseases and storage life of Embul banana. J. Horticultural Sci. Biotech. 8, (2003): 780-785.

2. Barnett BB and Hunter HL (1972) Illustrated genera of imperfect fungi. Burgess Publishing Co. Minneapolis, USA.

3. Bishop CD and Thornton IB. Evaluation of the antifungal activity of the essential oils of Monarda citriodora var. citriodora and Melaleuca alternifolia on the post harvest pathogens, Journal of Essential oil Research. 9, (1997): 77-82.

4. Curini, M., Bianchi, A., Epifano, F., Bruni, R., Torta, L., and Zambonelli, A. Composition and in vitro antifungal activity of essential oils of Erigeron Canadensis and Myrtus communis from France. Chemistry of Natural Compounds. 39, (2003): 2-9.

5. Deans SG and Ritchie G. Antimicrobial properties of plant essential oils. Int. J. Food Microbiol. 5, (1987): 165-180

6. Devi, S.A and Ganjewala, D. Antimicrobial activity of Acorus calamus (L.) rhizome and leaf extract. Acta Biologica szegediensis. 53, (2009): 45- 49.

7. Domsch KH, Games W and Anderson T (1980) Compendium of soil fungi. Academic Press, London.

8. Fawcett, C.H. and D.M. Spencer. Plant chemotherapy with natural products. Ann. Rev Phytopath. 8, (1970): 403-418.

9. Grover RK and Moore JD. Toximetric study of fungicides against brown rot organism, Sclerotinia fructicola and S. laxa. Phytopathol. 52, (1962): 876-880.

10. Guenther E (1972) The essential oils Vol.I and IV. Robert, E. Krieger publishing Co. Hutington, New York.

11. Johnson LE and Curl EA (1972) Methods for research on ecology of soil borne plant pathogens. Burgess Publishing Co. Minneapolis, USA.

12. Lee, S.O., Choi, G.J., Jang, K.S., Lim, H.K., Cho, K.Y and Kim, J.C. Antifungal activities of five plant essential oils as fumigant against postharvest and soilborne plant pathogenic fungi. Plant pathol. J. 23, (2007): 97-102. 
13. Mazza, G. Gas chromatographic and mass spectrometric studies of the constituents of the rhizome of calamus (Acorus calamus): 1. Volatile constituents of the essential oil. J. Chromatogr. 328, (1985):179-194.

14. Ormancey, X., Sisalli, S and Coutiere, P. Formulation of essential oils in functional perfumery. Parfums, Cosmetiques, Actualités. 157, (2001): 30-40.

15. Parajuli, D.P., A.R. Gyawali and B.M. Shrestha. (1998). A Manual of the Important Non-Timber Forest Products in Nepal. Training and manpower development in C.F.M. Pokhara, Nepal.

16. Perrucci, S., Mancianti, f., Ciont, PL., Flamini, G., Morelli, I and Macchioni, G. In vitro antifungal activity of essential oils against some isolates of Microsporum canis and M. gypseum. Planta.Medi. 60, (1994): 184-187.

17. Radušienè, J., Judžentienè, A., Pečiulyte, D and Janulis, V. Essential oil composition and antimicrobial assay of Acorus calamus leaves from different wild populations. Plant Genetic Resources 5, (2007): 37-44.

18. Reuveni, R, Fleischer A and Putievski, E. Fungistatic activity of essential oils from Ocimum basilicum Chemotypes.Phitopatol.Z. 10, (1984):20-22.

19. Sati, S.C., Sati, N., Ahluwalia, V., Walia, S and Sati, O.P. Chemical composition and antifungal activity of Artemisia nilagirica essential oil growing in northern hilly areas of India. Natural Product Research 27, (2012): 45-48.

20. Satyal P, Paudel P, Poudel A, Dosoky NS, Moriarity DM, Vogler B, Setzer WN. Chemical compositions, phytotoxicity, and biological activities of Acorus calamus essential oils from Nepal. Nat Prod Commun. 8, (2013): 79-81.

21. Sharma, P. K., Raina, A.P., and Dureja, P. Evaluation of the antifungal and phytotoxic effects of various essential oils against Sclerotium rolfsii
(Sacc) and Rhizoctonia bataticola (Taub). Phytopathology and plant protection 27, (2007):1-8.

22. Stappen I, Wanner J, Tabanca N, Wedge D.E, Ali A, Khan I.A, Kaul V.K, Lal B, Jaitak V, Gochev V, Girova T, Stoyanova A, Schmidt E, Jirovetz L. Chemical Composition and Biological Effects of Artemisia maritima and Artemisia nilagirica Essential Oils from Wild Plants of Western Himalaya. Planta Med. 80, (2014): 1079-87.

23. Thompson, DP. Fungitoxic activity of essential oil components on food storage fungi. Mycologia. 81, (1989): 151-153.

24. Tripathi P and Shukla AK. Emerging nonconvensional technologies for control of post harvest diseases of perishables. Fresh Produce. 1, (2007): 111-120.

25. Tripathi P, Dubey NK and Shukla AK. Use of some essential oils as post harvest botanical fungicides in the management of grey mould of grapes caused by Botrytis cinerea. World J Microbiol. Biotech. 24, (2008): 39-46.

26. Unnithan, C. R., Muuz, M., Woldu, A., Reddy, D.N., Gebremariam, G., Menasbo, B., Hilawie, M and Teklu, T. Chemical analysis of the essential oil of Erigeron canadensis L. Unique. Journal of Pharmaceutical and Biological Sciences 2, (2014): 8-10.

27. Wijesekara, R.O.B., Ratnatunga, C.M. and Durbeck, K. (1997). The distillation of essential oils. Manufacturing and plant constructions handbook. Escgborn, Federal Republic of Germany: Protrade, Department of Foodstuffs \& Agricultural products.

\section{Cite this article as:}

Habung Yami and A.K. Shukla. Antifungal activity of essential oils derived from some plants against phytopathogenic fungi. Annals of Plant Sciences 5.7 (2016): 1374-1380.

DOI:http://dx.doi.org/10.21746/aps.2016.07.002
Source of support: Nil

Conflict of interest: None Declared 